\title{
Asymmetrical Naproxen-Conjugated Dendrimer for Targeted- Drug Delivery to Human Prostatic Adenocarcinoma Cancer Cells
}

\author{
Ulises Organista-Mateos ${ }^{1}$, Luis D. Pedro-Hernández ${ }^{1}$, Elena Martínez-Klimova ${ }^{2}$, Sandra Cortez-Maya ${ }^{1}$, Teresa Ramírez-Ápan ${ }^{1}$ and \\ Marcos Martínez-García ${ }^{1 *}$ \\ ${ }^{1}$ Instituto de Química, Universidad Nacional Autónoma de México, Ciudad Universitaria, Circuito Exterior, Coyoacán, C.P. 04510, México D.F., México \\ ${ }^{2}$ Facultad de Química, Universidad Nacional Autónoma de México, Ciudad Universitaria, Circuito Interior, Coyoacán, C.P. 04510, México D.F., México \\ *Corresponding author: Dr. Marcos Martínez-García, Instituto de Química, Universidad Nacional Autónoma de México, Ciudad Universitaria, Circuito Exterior, Coyoacán, \\ C.P. 04510, México D.F., México; Fax: +52+55+5616-22-03; Email: margar@unam.mx
}

Received: June 25, 2019; Accepted: July 05, 2019; Published: July 09 2019;

\begin{abstract}
Naproxen was directly conjugated to $\mathrm{NH}_{2}$-terminated dendrimers by an amide bond and OH-terminated dendrimers by an ester bond. The drugconjugated polyamidoamine dendrimers showed better cellular uptake than free naproxen. Free naproxen and conjugates in vitro cytotoxicity studies were performed in U251, PC3, K-562, HCT-15, MCF-7 and SKLU-1 cancer cells using different cytotoxicity assays. Naproxen-conjugates of first and second generation showed significant cytotoxic effects in human prostatic adenocarcinoma PC-3 and human mammary adenocarcinoma MCF-7. Moreover, the naproxen-conjugates improved cytotoxicity compared to free naproxen. The increased therapeutic efficacy was observed in specific naproxen conjugates of first generation using low doses, demonstrating that the conjugate was as potent as the antiproliferative agent cisplatin.
\end{abstract}

Keywords: Polyamidoamine-dendrimers, Naproxen-conjugates, Anti-cancer activity.

\section{Introduction}

Dendrimers are homogeneous structures from their nucleus to their terminal group, and also in their internal branching moieties. Dendrimers have unique properties, such as monomolecular weight and good biocompatibility. Dendrimers are spherical threedimensional structures with exchanged surface functionalities [1-3]. Dendrimers have a great potential for applications in many fields, including drug/gene delivery, as catalysts, and in bioimaging. Among these, applications have been a key focus of research in nanotechnology and nanobiology [4,5]. Bioactive compounds could be joined with the dendrimers and can act as efficient nanocarriers of the bioactive compounds [6]. Naproxen (Nap, see Scheme 2) is a member of the class of Non-steroidal Anti-Inflammatory Drugs (NSAIDs). As one of the most commonly-used cyclooxygenase (COX) inhibitors, it has been used for the treatment of many inflammationassociated conditions, e.g. arthritis, gout, tendinitis, and bursitis $[7,8]$. Although some COX-2 inhibitors were demonstrated to be related to increases in the risk of cardiovascular events and gastrointestinal adverse effects [9-11], an emerging body of data suggests that Nap rarely increases the risk of adverse cardiovascular events compared to other NSAIDs. In the literature, the synthesis of dendrimers with naproxen as conjugates or complexes has been reported, improving the solubility and the permeability of the drug. It was also was found that the conjugates of naproxen showed anticancer activity [7-13]. Recently, we designed and synthesized several types of NSAIDs- modified dendrimers with good drug-delivery properties [12,13]. Herein we have further designed and developed naproxen and polyamidoamine-modified dendrimers as possible targeted drug carriers towards human prostatic adenocarcinoma and human mammary adenocarcinoma cancer cells.

\section{Materials and Methods}

${ }^{1} \mathrm{H}$ and ${ }^{13} \mathrm{C}$ NMR spectra were recorded on a Varian Unity-300 $\mathrm{MHz}$ with Tetramethylsilane (TMS) as an internal reference. Infrared (IR) spectra were measured on a Nicolet FT-SSX spectrophotometer. Elemental analysis was determined by Galbraith Laboratories, INC Knoxville. FAB+ mass spectra were taken on a JEOL JMS AX505 HA instrument. Electrospray mass spectra were taken on a Bruker Daltonic, Esquire 6000. MALDI-TOF mass spectra were taken on a Bruker Omni FLEX using 9-nitroanthracene (9NA) as a matrix. The UV-vis absorption spectra were obtained at room temperature with a Shimadzu 2401 PC spectrophotometer.

\section{Characterization of the Conjugates}

${ }^{1} \mathrm{H}$ and ${ }^{13} \mathrm{C}$ NMR spectra were recorded on a Varian Unity-300 $\mathrm{MHz}$ with tetramethylsilane (TMS) as an internal reference. Infrared (IR) spectra were measured on a Nicolet FT-SSX spectrophotometer. Elemental analysis was determined by Galbraith Laboratories, INC Knoxville. FAB+ mass spectra were taken on a JEOL JMS AX505 HA instrument. Electrospray mass spectra were taken on a Bruker 
Daltonic, Esquire 6000. MALDI-TOF mass spectra were taken on a Bruker Omni FLEX using 9-nitroanthracene (9NA) as a matrix. The UV-vis absorption spectra were obtained at room temperature with a Shimadzu 2401 PC spectrophotometer.

\section{Anticancer Screening}

U-251 (human glioblastoma), PC-3 (human prostatic adenocarcinoma), K-562 (human chronic myelogenous leukemia cells), HCT-15 (human colorectal adenocarcinoma), MCF-7 (human mammary adenocarcinoma), SKLU-1 (human lung adenocarcinoma) cell lines were supplied by the National Cancer Institute (USA). COS 7 Monkey African green kidney, SV40 transformed cells were supplied by National Cancer Institute (USA). Cytotoxicity assays were determined using the protein-binding dye sulforhodamine B (SRB) in microculture to measure cell growth, as described $[12,13]$. Conjugates of naproxen and polyamidoamine dendrimers were prepared in 2
$\%$ DMSO and added into the culture medium immediately before use. Control cells were treated with $2 \%$ DMSO. For the assay with COS 7 Monkey African green kidney, SV40 transformed, the tested compounds were dissolved in fresh culture medium with $2 \%$ DMSO to afford different concentrations $(1,10,50,100 \mu \mathrm{mol} / \mathrm{L})$.

\section{Results and Discussion}

\section{Synthesis of Ethanolamine Polyaminoamide Dendritic Arms Dendrimers}

The PAMAM dendrimers were synthesized by a divergent approach using ethanolamine as the core. This methodology involves typical stepwise and iterative two-step reaction sequences, consisting of the Michael addition of primary amines with methyl acrylate and the amidation of methyl ester groups with ethylendiamine to produce amine terminations (Scheme 1).

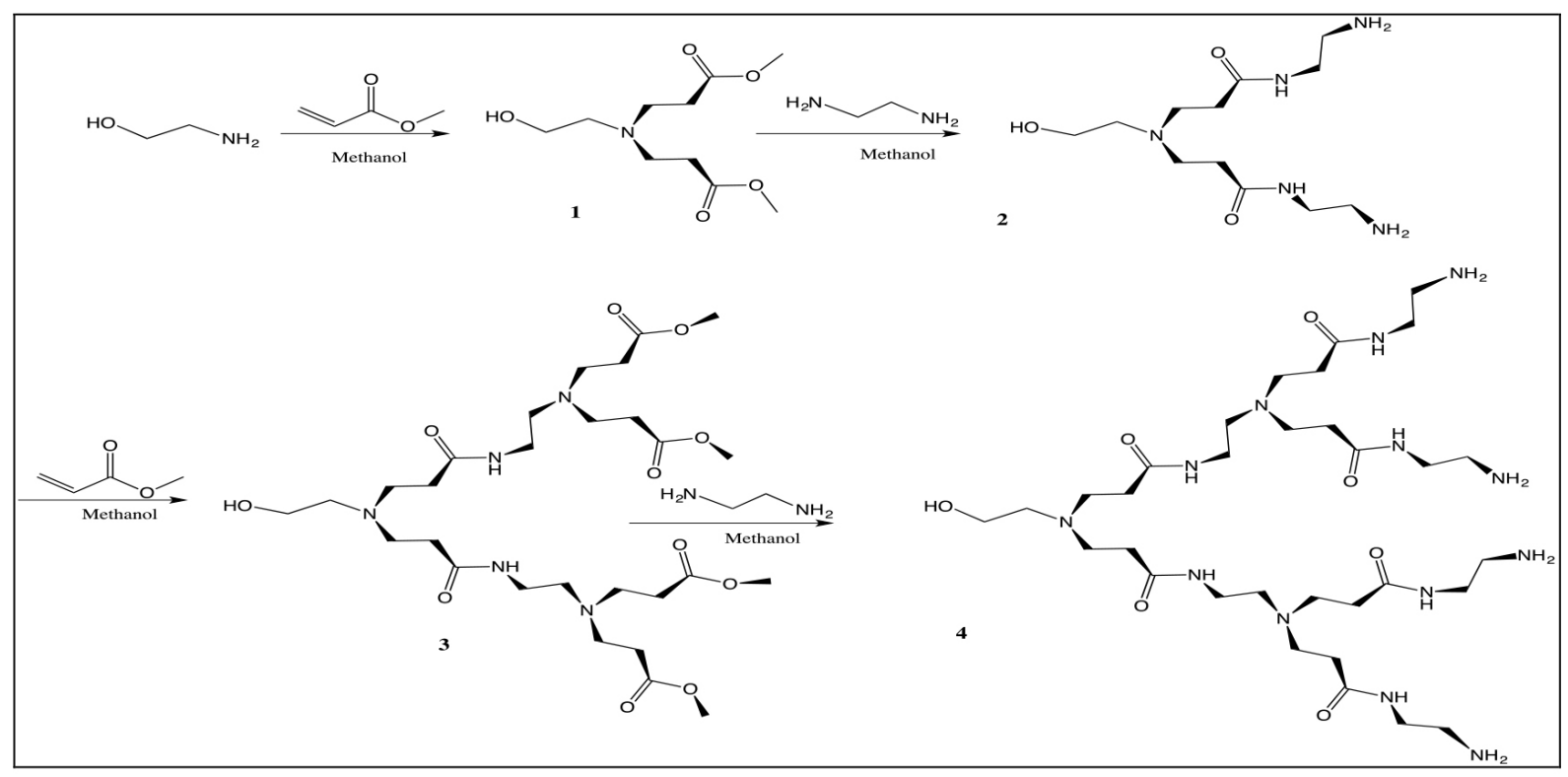

Scheme 1. Synthesis of the ethanolamine PAMAM-dendrimers

The dendrons of first and second generation were characterized by ${ }^{1} \mathrm{H},{ }^{13} \mathrm{C}$ NMR, FTIR, UV-Vis spectroscopy and mass spectrometry.

\section{Synthesis of Naproxen Chloride}

After that, from the naproxen acid, the naproxen chloride with thionyl chloride in $\mathrm{CH}_{2} \mathrm{Cl}_{2}$ at reflux for $3 \mathrm{~h}$ was obtained (Scheme 2) and characterized by ${ }^{1} \mathrm{H},{ }^{13} \mathrm{C}$ NMR and mass spectrometry.

\section{Synthesis of Naproxen Dendrimers}

The naproxen chloride was coupled to the dendrons with ethanolamine and amine terminal groups (Chart 1). For the NMR spectra of compounds 7 and 8, methanol deuterated was used as solvent. Their peaks were similar to those of compounds $\mathbf{2}$ and $\mathbf{4}$. The high-resolution mass spectra results of compounds 7 and 8 were 926.4 and $1806.8 \mathrm{~m} / \mathrm{z}$, respectively.
In the ${ }^{1} \mathrm{H}$ NMR spectra, the following signals were observed: at $\delta_{\mathrm{H}} 8.26$ one broad signal due to the $\mathrm{NH}$ groups, three broad signals at $\delta_{\mathrm{H}} 7.73-7.12$ assigned to the Ar protons from naproxen, one singlet at $\delta_{\mathrm{H}} 3.83$ for the $\mathrm{OCH}_{3}$ groups, one broad signal at $\delta_{\mathrm{H}} 3.75$ for the $\mathrm{CH}$ protons due from the naproxen moiety, three broad signals at $\delta_{\mathrm{H}} 3.38-$ 2.24 due to the dendritic branches and finally at $\delta_{\mathrm{H}} 1.39$, one broad signal for the $\mathrm{CH}_{3}$ from the naproxen moiety.

\section{Cytotoxicity of Naproxen conjugates}

The cytotoxic activity of the synthesized PAMAM-ethanol derivatives 3, 4 and the conjugate compounds of naproxen 7 and 8 were chosen for evaluation of their biological activity against cancer cell lines. We screened in vitro against seven human cancer cell lines: U251 (human glioblastoma), PC-3 (human prostatic adenocarcinoma), K-562 (human chronic myelogenous leukemia 
cells), HCT-15 (human colorectal adenocarcinoma), MCF-7 (human mammary adenocarcinoma), SKLU-1 (human lung adenocarcinoma). As a control, we also tested against the COS-7 African green monkey kidney cell line. The free naproxen and cisplatin were used to compare the antiproliferative activity of the dendrimers 3,4 and 7,8 . The dendrimers 3 and $\mathbf{4}$ with two and four $\mathrm{NH}_{2}$ terminal groups at 10 $\mu \mathrm{M}$ showed low anticancer activity against all the cells used. In the case of the conjugates $\mathbf{7}$ and $\mathbf{8}$, the concentration of the conjugate was diluted in 3 and 5 times to have the anticancer activity for one molecule of naproxen and compare it to the free naproxen. Table 1 shows the normalized percentage of inhibition of the growth that allows comparing the activity of the same amount of naproxen in its free state and when it is contained in the conjugates of compounds 7 and 8.

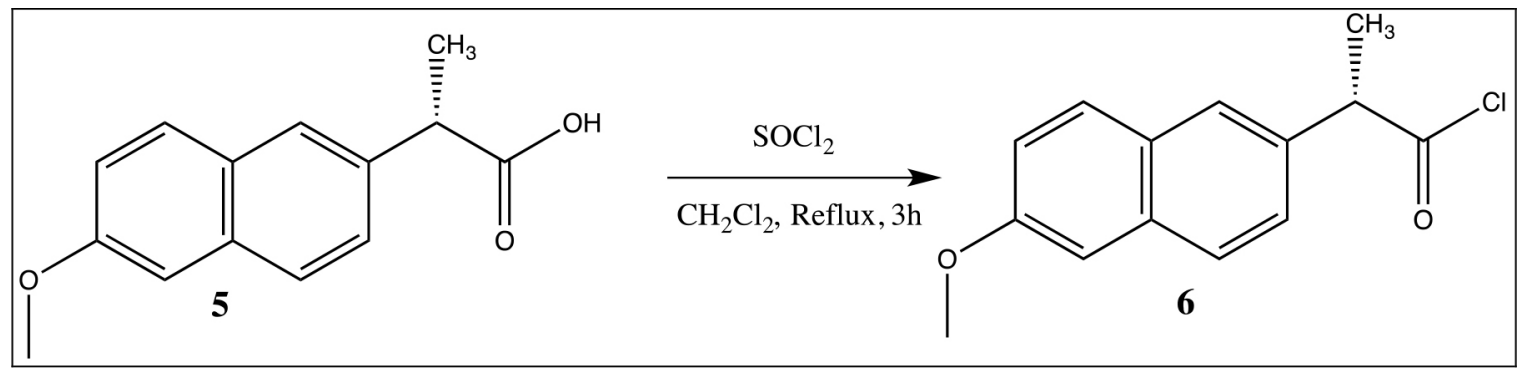

Scheme 2. Synthesis of the naproxen chloride $\mathbf{1 3}$

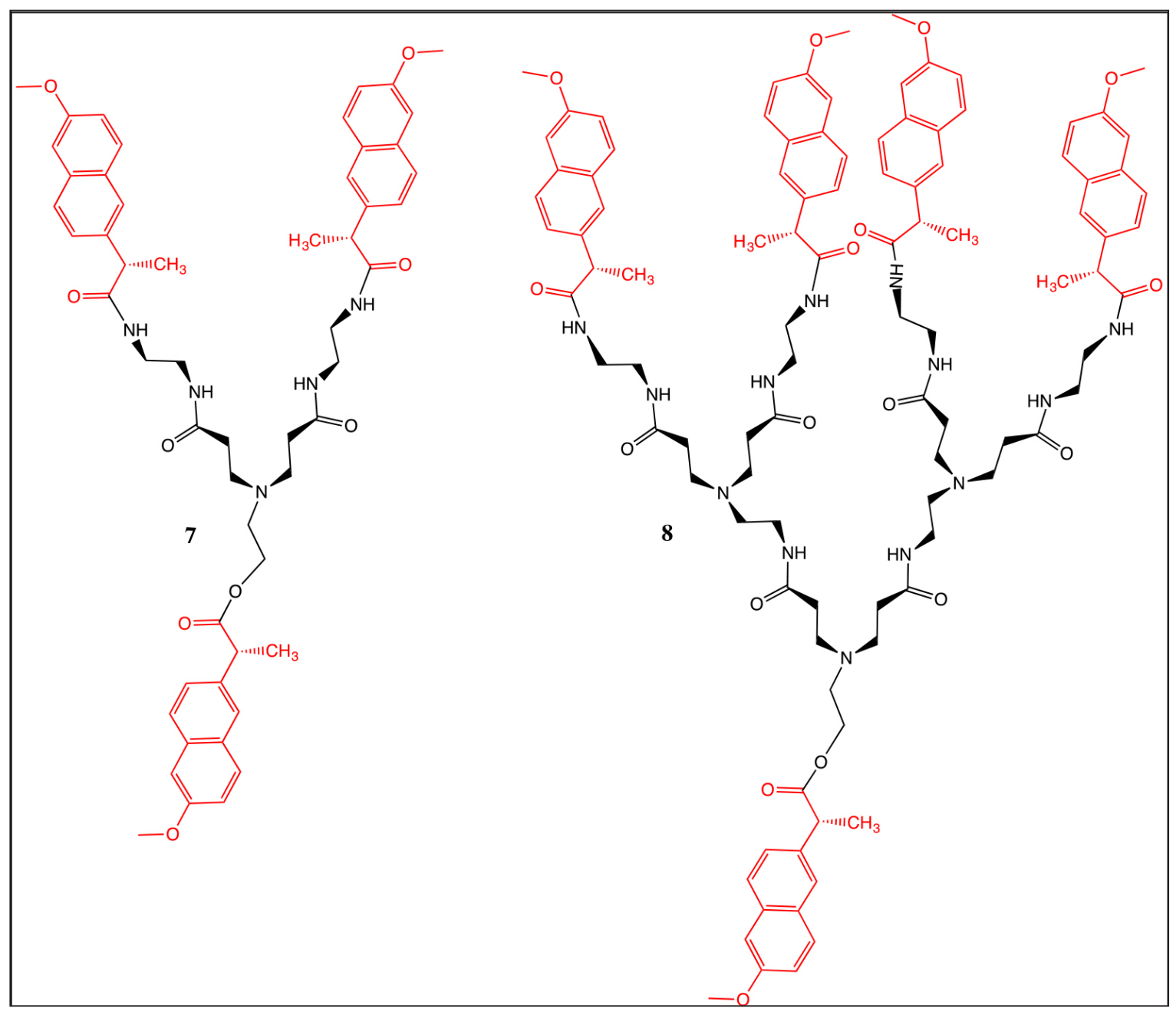

Chart 1. Naproxen derivatives of first $\mathbf{7}$ and second $\mathbf{8}$ generation. 
Table 1. Cytotoxic activity of the compounds $3,4,7$ and 8 at $10 \mu \mathrm{M}$.

\begin{tabular}{|l|c|c|c|c|c|c|c|}
\hline & \multicolumn{7}{|c|}{ \% of inhibitión } \\
\hline Sample & U251 & PC-3 & K562 & HCT-15 & MCF-7 & SKLU-1 & COS7 \\
\hline $\mathbf{3}$ & $\mathrm{NC}$ & $1.8 \pm 2.2$ & $1.6 \pm 1.1$ & $\mathrm{NC}$ & $1.8 \pm 1.2$ & $\mathrm{NC}$ & $\mathrm{NC}$ \\
\hline $\mathbf{4}$ & $\mathrm{NC}$ & $1.2 \pm 1.7$ & $1.9 \pm 1.2$ & $0.6 \pm 0.6$ & $1.1 \pm 1.3$ & $\mathrm{NC}$ & $\mathrm{NC}$ \\
\hline $\mathbf{7}$ & $4.5 \pm 1.4$ & $53.0 \pm 2.2$ & $6 \pm 1.5$ & $7.5 \pm 0.8$ & $33.0 \pm 1.0$ & $11.1 \pm 1.3$ & $\mathrm{NC}$ \\
\hline $\mathbf{8}$ & $5.1 \pm 1.1$ & $59.4 \pm 1.1$ & $7.1 \pm 1.4$ & $8.1 \pm 1.3$ & $38.5 \pm 1.9$ & $15.3 \pm 0.8$ & $\mathrm{NC}$ \\
\hline Naproxen & $\mathrm{NC}$ & $\mathrm{NC}$ & 1.76 & 2.23 & 6.43 & $\mathrm{NC}$ & $\mathrm{NC}$ \\
\hline Cisplatin & 87.49 & 42.65 & 79.15 & 32.42 & 32.42 & 81.35 & 42.39 \\
\hline
\end{tabular}

$\mathrm{NC}=$ non cytotoxic

Cisplatin was tested at the same concentration of $10 \mu \mathrm{M}$. The initially obtained cytotoxic screening data (Table 1) showed that at $10 \mu \mathrm{M}$, the conjugate 7 showed good inhibition activity $53.0 \pm 2.2$ (\%) against the human prostatic adenocarcinoma PC-3 cell line and $33.0 \pm 1.0 \%$ against human mammary adenocarcinoma MCF-7 cell line, this activity was higher than the free naproxen. The conjugate 8 showed better activity from $15.3 \pm 0.8$ to $39.4 \pm 1.1$ against PC-3, MCF-7 SKLU-1 in comparison with the naproxen alone. For the dendrimer conjugate naproxen 7 the activity against PC-3 and MCF-7 was very close to that of cisplatin. The antiproliferative results obtained with the conjugates of naproxen $\mathbf{7}$ and $\mathbf{8}$ were compared to cisplatin, as a reference anticancer drug. The compounds 3, 4 and the conjugates $\mathbf{7}$ and $\mathbf{8}$ did not show any activity against the COS-7 African green monkey kidney cell line.

\section{Conclusion}

Naproxen was directly conjugated to $\mathrm{NH}_{2}$-terminated dendrimers by an amide bond and $\mathrm{OH}$-terminated dendrimers by an ester bond. The drug-conjugated polyamidoamine dendrimers showed better cellular uptake than free naproxen. Free naproxen and conjugates' in vitro cytotoxicity studies were performed in U251, PC3, K-562, HCT15, MCF-7 and SKLU-1 cancer cells using different cytotoxicity assays. Naproxen-conjugates of first and second generation showed significant cytotoxic effects in human prostatic adenocarcinoma PC-3 and human mammary adenocarcinoma MCF-7. Moreover, the naproxenconjugates improved cytotoxicity compared to the free naproxen. The increased therapeutic efficacy was observed in specific naproxen conjugates of first generation using low doses demonstrating that the conjugate was equally potent as the antiproliferative agent cisplatin.

\section{Supplementary Material}

Supplementary material is associated with this manuscript. It contains general experimental procedures, compound characterization data, and copies of ${ }^{1} \mathrm{H}$ and ${ }^{13} \mathrm{C}$ NMR spectra of representative compounds.

\section{Acknowledgment}

This work was supported by DGAPA IN101117 grants. EMK was supported by a postdoctoral fellowship from CONACyT-México. We would also like to thank Rios O.H., Velasco L., Huerta S.E., Patiño M.M.R., Peña Gonzalez M.A., Rios Ruiz L. and Garcia Rios E. for technical assistance.

\section{References}

1. Sadekar S, Ghandehar H (2012) Transepithelial transport and toxicity of PAMAM dendrimers: Implications for oral drug delivery. Adv Drug Delivery Rev 64: 571-88.

2. Mignani S, El Kazzouli S, Bousmina M, Majoral JP (2013) Expand classical drug administration ways by emerging routes using dendrimer drug delivery systems: A concise overview. Adv Drug Delivery Rev 65: 1316-30.

3. Kesharwani P, Jain K, Kumar NJ (2014) Dendrimer as nanocarrier for drug delivery Progress in Polymer Science. 39: 268-307.

4. Huang D, Wub D (2018) Biodegradable dendrimers for drug delivery. Materials Science \& Engineering 90: 713-27.

5. Sherje AP, Jadhav M, Dravyakar BR, Kadam D (2018) Dendrimers: A versatile nanocarrier for drug delivery and targeting. Inter J of Pharmaceutics 548: 707-20

6. Kesharwani P, Gothwal A, Iyer AK, Jain K, Chourasia MK, Gupta U (2018) Dendrimer nanohybrid carrier systems: an expanding horizon for targeted drug and gene delivery. Drug Discovery Today 23:300-14.

7. Zhao Y, Zeng Q, Wu F, Li J, Pan Z, et al (2016) Novel naproxen-peptide-conjugated amphiphilic dendrimer self-assembly micelles for targeting drug delivery to osteosarcoma cells. RSC Adv 6: 60327-60335

8. Gasparini G, Longo R, Sarmiento R, Morabito A (2003) Inhibitors of cyclooxygenase 2: a new class of anticancer agents? Lancet Oncol 4: 605-615.

9. Najlah M, Freeman S, Attwood D, D'Emanuele A (2007) In vitro evaluation of dendrimer prodrugs for oral drug delivery. Inter J of Pharmaceutics 336:183-90.

10. Najlah M, Freeman S, Khoder M, Attwood D, D'Emanuele A (2017) In Vitro Evaluation of Third Generation PAMAM Dendrimer Conjugates. Molecules 22:1661-1673.

11. Ouyanga L, Mac L, Lia Y, Pana J, Guoa L (2010) Synthesis of water-soluble first generation Janus-Type dendrimers bearing Asp oligopeptides and naproxen. ARKIVOC: 256-66

12. Padilla-Monroy S, Martínez-Klimova E, Ramírez-Ápan T, Nieto-Camacho A, Calderón-Pardo J, et al (2018) Porphyrin Conjugates of Ibuprofen and their Antiproliferative Activity Against Human Prostate and Breast Cancer Cells. Biointerface Research in Applied Chemistry 8: 3039-48.

13. Pedro-Hernández LD, Martínez-Klimova E, Cortez-Maya S, Mendoza-Cardozo S, Ramírez-Ápan T, et al (2017) Synthesis, Characterization and Nanomedical Applications of Conjugates Between resorcinarene-Dendrimers and Ibuprofen. Nanomaterials 7: 163-73.

\section{Citation:}

Organista-Mateos U, Pedro-Hernández LD, Martínez-Klimova E, Cortez-Maya S, Ramírez-Ápan T, Martínez-García M (2019) Asymmetrical NaproxenConjugated Dendrimer for Targeted-Drug Delivery to Human Prostatic Adenocarcinoma Cancer Cells. J Pharmacol Pharm Res Volume 2 (3): 1-4. 